\title{
Validating the correct wearing of protection mask by taking a selfie: design of a mobile application "CheckYourMask" to limit the spread of COVID-19
}

This paper was downloaded from TechRxiv (https://www.techrxiv.org).

\section{LICENSE}

CC BY 4.0

SUBMISSION DATE / POSTED DATE

$22-05-2020$ / 28-05-2020

\section{CITATION}

Hammoudi, Karim; Cabani, Adnane; Benhabiles, Halim; Melkemi, Mahmoud (2020): Validating the correct wearing of protection mask by taking a selfie: design of a mobile application "CheckYourMask" to limit the spread of COVID-19. TechRxiv. Preprint. https://doi.org/10.36227/techrxiv.12355970.v1

\section{$\mathrm{DOI}$}




\section{Validating the correct wearing of protection mask by taking a selfie: design of a mobile application "CheckYourMask" to limit the spread of COVID-19}

\author{
Karim Hammoudi ${ }^{1,2, *}$ \\ ${ }^{1}$ Université de Haute-Alsace \\ Department of Computer Science, IRIMAS \\ F-68100 Mulhouse, France \\ ${ }^{2}$ Université de Strasbourg \\ ${ }^{*}$ Corresponding author \\ karim.hammoudi@uha.fr
}

\author{
Mahmoud Melkemi ${ }^{1,2}$ \\ ${ }^{1}$ Université de Haute-Alsace \\ Department of Computer Science, IRIMAS \\ F-68100 Mulhouse, France \\ ${ }^{2}$ Université de Strasbourg \\ mahmoud.melkemi@uha.fr
}

\author{
Adnane Cabani ${ }^{3}$ \\ ${ }^{3}$ Normandie Univ, UNIROUEN \\ ESIGELEC, IRSEEM \\ 76000 Rouen, France \\ adnane.cabani@esigelec.fr
}

\author{
Halim Benhabiles ${ }^{4}$ \\ ${ }^{4}$ Univ. Lille, CNRS, Centrale Lille \\ Univ. Polytechnique Hauts-de-France \\ Yncrea Hauts-de-France \\ UMR 8520 - IEMN \\ Lille F-59000, France \\ halim.benhabiles@yncrea.fr
}

\begin{abstract}
In a context of a virus that is transmissive by sputtering, wearing masks appear necessary to protect the wearer and to limit the propagation of the disease. Currently, we are facing the 2019-20 coronavirus pandemic. Coronavirus disease 2019 (COVID-19) is an infectious disease with first symptoms similar to the flu. COVID-19 appeared first in China and very quickly spreads to the rest of the world. The COVID-19 contagiousness is known to be high by comparison with the flu. In this paper, we propose a design of a mobile application for permitting to everyone having a smartphone and being able to take a picture to verify that his/her protection mask is correctly positioned on his/her face. Such application can be particularly useful for people using face protection mask for the first time and notably for children and old people. The designed method exploits Haar-like feature descriptors to detect key features of the face and a decision-making algorithm is applied. Experimental results show the potential of this method in the validation of the correct mask wearing. To the best of our knowledge, our work is the only one that currently proposes a mobile application design "CheckYourMask" for validating the correct wearing of protection mask.

Index Terms-Face protection masks, public health support, health education, COVID-19, coronavirus, e-health, m-health, mobile health, public health system, epidemic prevention and control.
\end{abstract}

\section{INTRODUCTION AND MOTIVATION}

In the context of transmitted virus between humans by sputtering (spraying), wearing the mask on the face appears necessary to protect people and to limit the propagation of the disease. Currently, we are facing the 2019-20 coronavirus pandemic. Coronavirus disease 2019 (COVID-19) is an infectious disease with first symptoms similar to the flu. COVID-19 appeared first in China and very quickly spreads to the rest of the world. The COVID-19 contagiousness is known to be high

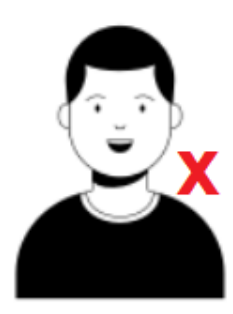

(a) no mask

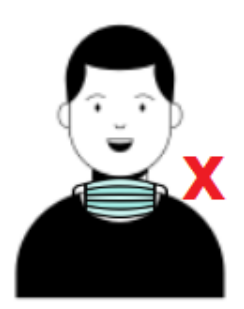

(d) unprotected

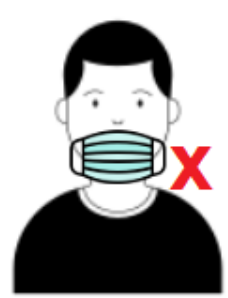

(b) unprotected nose

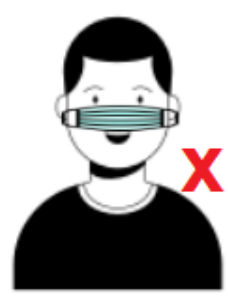

(e) unprotected chin and mouth

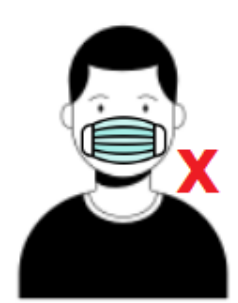

(c) unprotected chin

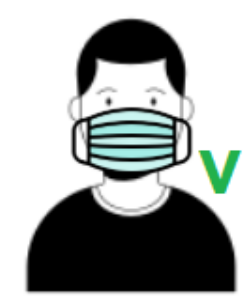

(f) protected nose, mouth and chin
Fig. 1: Various configurations related to the mask wearing.

by comparison with the flu. In this paper, we propose a design of a mobile application allowing people able to take a picture with a smartphone to verify that his/her protection mask is correctly positioned on his/her face. Such application can be particularly useful for people using face protection mask for the first time and notably for children and old people.

The contagiousness of COVID-19 is discussed in [1]. Authors show Figures that permits to become aware about the interest of face mask wearing in the COVID-19 context. In particular, the Figure on Page 5 of their article illustrates the 
distance of virus dispersion according to postilions, sneezing or coughing and the Figure on Page 7 illustrates the risks of contamination according to different face-to-face scenarios with mask wearing and/or not wearing. For this latter, 4 levels of risks are represented and described.

Although many people are already convinced of the interest for wearing face protection mask such as suggested by the World Health Organization [2] and scientific studies [3]-[5], one can observe that many individuals do not correctly wear their masks (see various mask wearing configurations in Figure 11. These observations have led nurses and other citizens to initiate prevention campaigns related to the public health education in wearing the mask. Precisely, these campaigns consist of sensitizing people about the correct and incorrect manner to wear face protection masks by disseminating prevention posters and drawings [6]-[9]. In our case, we propose to support these public health campaigns by designing an imagebased analysis method and an associated digital tool that are dedicated to the verification of the correct mask wearing by exploiting a smartphone and its frontal camera.

In the literature, some research works study the mobile phone data such as positioning data to carry out contact tracing in relation to COVID-19 [10]-[12]. People can receive information about those that have been infected and near them. They can also alert the connected people if they are themselves infected. This can permit connected people to get COVID-19 maps and to avoid areas with high risks of contamination.

Besides, the detection of mask through camera acquisition systems has also been investigated. In this context, applications are developed for detecting the presence of mask or not [13], for the counting of individual wearing mask towards carrying out crowd statistics and even for facial identification of people wearing mask [14]. Most of research systems in favor of the fight against COVID-19 are focused on people monitoring.

In our case, investigations directly deal with health education and take place upstream to limit the spread of COVID-19 through a m-health (mobile health) technology. To the best of our knowledge, our work is the only one that currently proposes a mobile application design "CheckYourMask" for validating the correct wearing of protection mask.

\section{Proposed FACE-FEATURE BASED METHOd TO VALIDATE THE WEARING OF THE MASK}

The designed preliminary method exploits Haar-like feature descriptors to detect the face as well as key features of the face from the camera-based acquisition of a mobile phone; namely e.g. detection of eyes, mouth, nose. It is assumed that a video selfie is taken when the camera is frontally facing the face for facilitating the face detection. Then a decisionmaking algorithm is applied from the detected face features (see basis Algorithm 11). In this version of the algorithm, if a face is detected, then it is assumed that the mask wearing is valid if the eyes are detected and if at the same time the detection of the nose, the mouth and the chin fail. Otherwise, it is concluded that the mask wearing is not valid. It is assumed that the method analyzes faces wearing conventional masks;

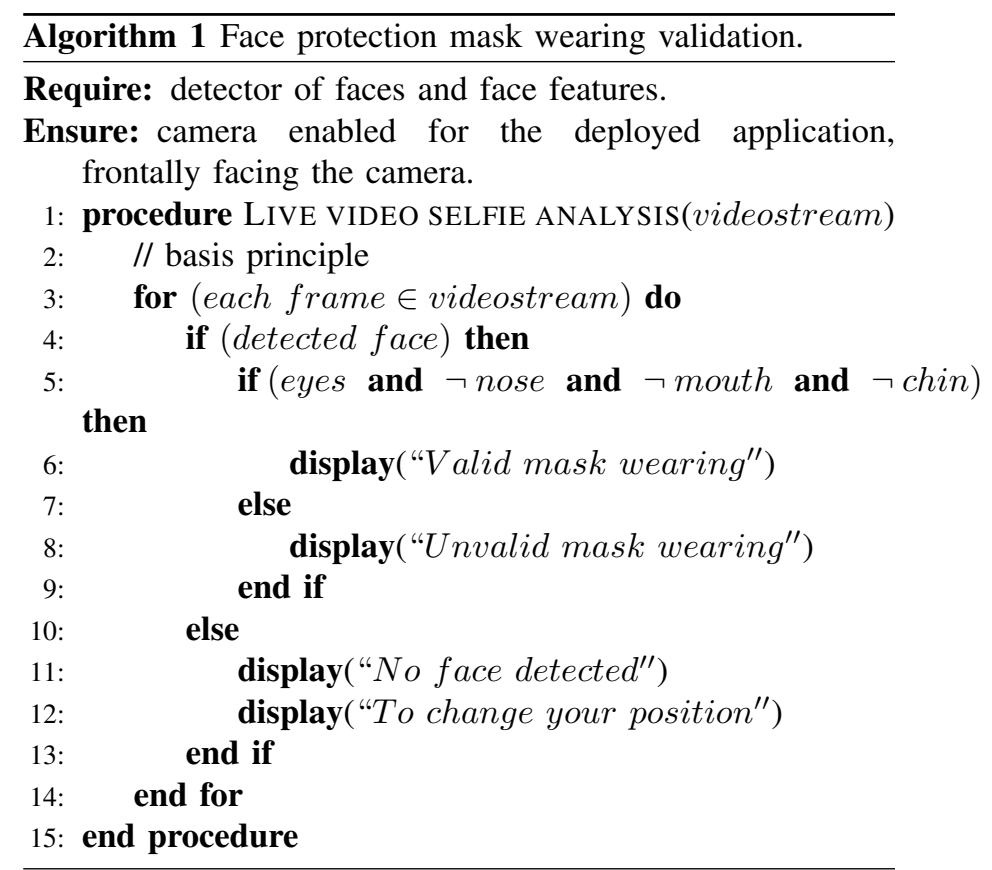

namely with an unique and opaque color. The presented preliminary mask wearing validation system aims to manage all mask wearing configuration of Figure 1 .

The basis principle of the algorithm notably exploits the real-time detection of multiple faces at different resolutions in video streams [15] and Haar feature-based cascade classifiers that rely on [16], [17].

\section{EXPERIMENTAL STUDY}

Experiments have been conducted by exploiting resources in design engineering and in image analysis accessible in Android and OpenCV environments. We observe that the use of Haar-like feature descriptors for detecting face and face features is sensitive. Indeed, various false detections can occur according to the condition of illumination during the acquisition, and to objects or textures that are visible out of the face. For this reason, the Algorithm 1 has been modified by limiting the detection of face features to the nose for preliminary experiments (see Algorithm 2).

The nominal outputs of the designed "CheckYourMask" application are displayed in Figure 2. In Subfigure 2a, the application asks to the user to change his position since no face has been detected. Subfigure $2 \mathrm{~b}$ shows that the face is detected. Since the nose is also detected, then it displays that the mask wearing is not valid. Subfigure $2 \mathrm{c}$ displays a user view for a similar scenario (nose rectangle is hidden). Subfigure $2 \mathrm{~d}$ displays the obtained outputs when the face is detected and the nose is not detected; namely, mask wearing is valid. The tested mask is a single-use face mask (brand "BYD Electronics").

Figure 3 shows a test of configurations such as shown in Figure 1. Scenario of Subfigure 1 a) is correctly obtained (see Subfigure 3a. The scenarios from Subfigures 1,b) to 1 (c) cannot be operated by using the considered white tissue mask since it is very rigid and since its ties are short. The scenario 


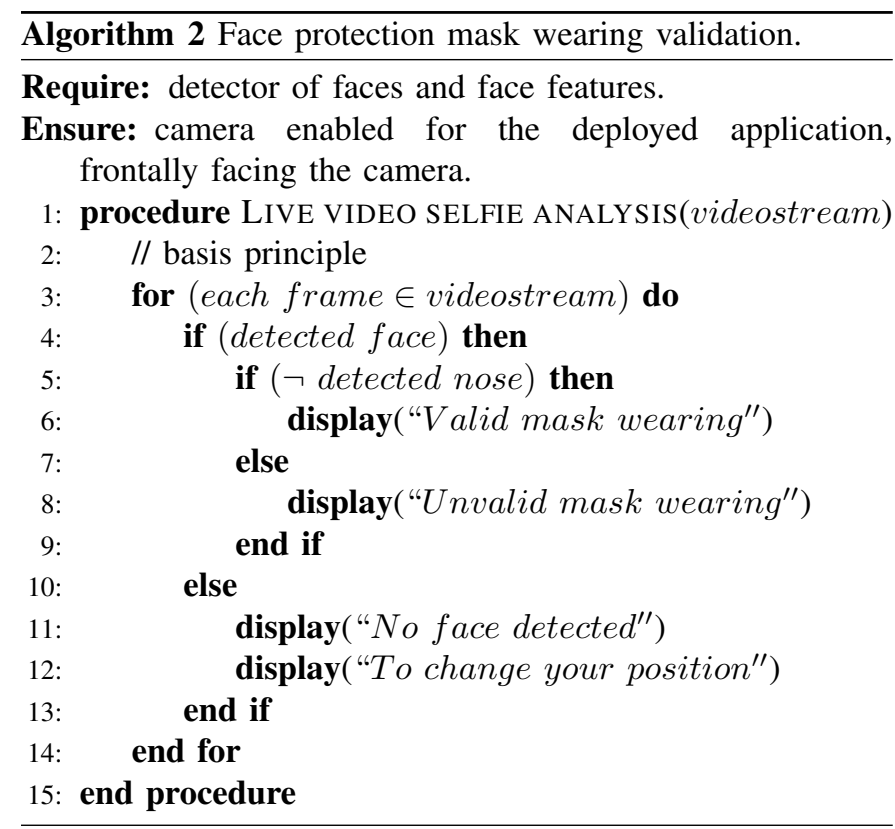

of 1(f) relatively works (see Subfigure 3c) except when the scene illumination is low. For this latter case, it may occur that the nose detector fails by surrounding another image region although the nose is hidden by a mask (see Subfigure 3b).

Figure 4 shows a test of configurations such as shown in Figure 1 by using a single-use blue face protection mask. For several scenarios, the face is not detected (Subfigures $4 \mathrm{a} 4 \mathrm{c} 4 \mathrm{f} / \mathrm{h}$ ). This can occur when a too large surface of the face is hidden by the mask. Besides, since this mask is relatively flexible, it fits the shape of the nose. Hence, it can cause nose detection (i.e., erroneous interpretation) even when the mask is weared such as shown in Subfigure 4d. The detection of the nose, and in particular of the face works when the mask is positioned on the neck and at the boundary of the face (see Subfigure 4e).

Figure 5 shows tests of the designed "CheckYourMask" application carried out by some users having masks of different natures; e.g. disposable and reusable, with dark and light colors (see Subfigures $5 \mathrm{~b} 5 \mathrm{~d}$ and $5 \mathrm{EF}$ (5h. respectively). Nominal detections are displayed with low and high image resolutions (e.g., see Subfigures 5f] and 5h, respectively).

\section{CONCLUSION AND FUTURE WORKS}

A method is designed for checking the correct wearing of face protection mask from a video selfie. Different analysis scenarios have been experimented using diverse types of conventional mask and varied acquisition conditions. The performance of the designed method relies on the efficiency of the exploited face and face-feature detectors. In the present study, wearing glasses had no negative effect. The use of rigid masks seems preferable because they reduce possibilities of wrong positioning on the face. For this latter, the designed prototype can particularly be efficient. Hence, a promising application "CheckYourMask" has been proposed. A proof of concept as well as a development base are provided towards

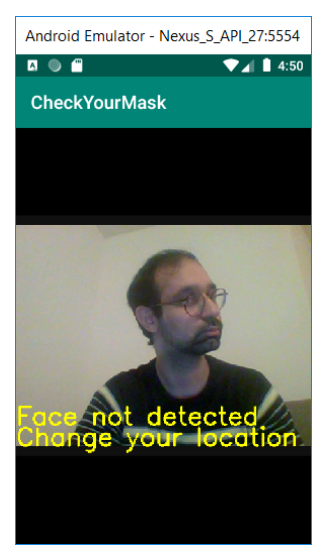

(a) Undetected face.

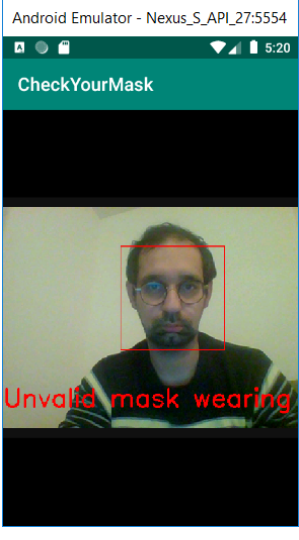

(c) User view (unvalid).

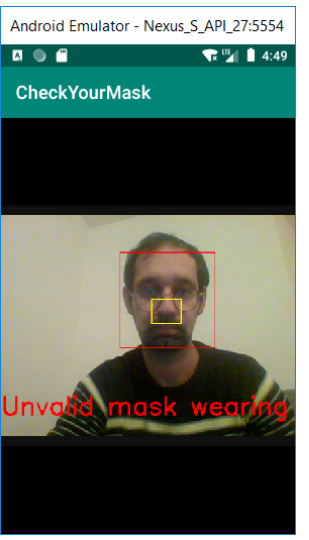

(b) Unvalid mask wearing.

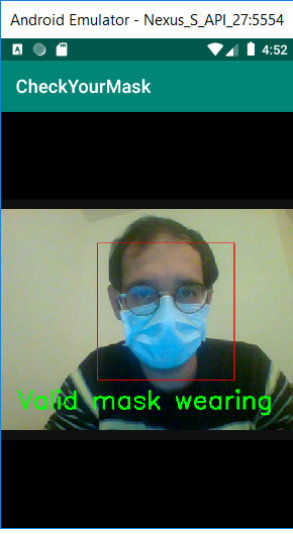

(d) Valid mask wearing.
Fig. 2: Different outputs of the "CheckYourMask" application. A single-use blue face protection mask is used.

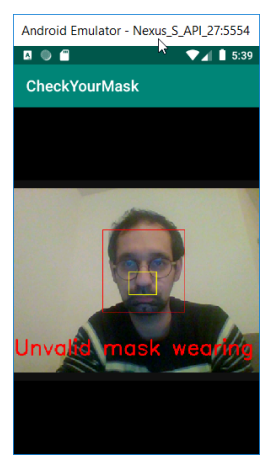

(a) Unvalid wearing.

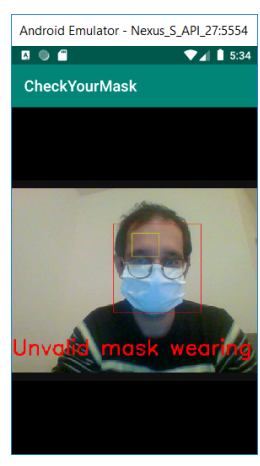

(b) Erroneous case.

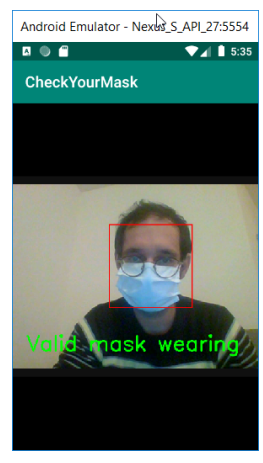

(c) Valid wearing
Fig. 3: Test of configurations with respect to Figure 1. The user wears glasses. The image background is homogeneous. A reusable white face protection mask made in tissue is used.

reducing the spread of COVID-19 by allowing people to validate the wearing of their mask via their smartphone (m-health). Moreover, this self-checking of the mask wearing could be exploited by monitoring-related applications as a conformity attribute of mask wearing. Future works may investigate the development of highly robust detectors by training a deep 


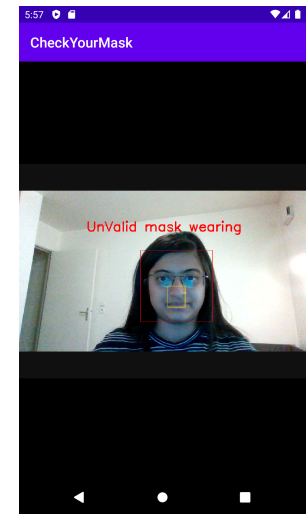

(a) Unvalid wearing.

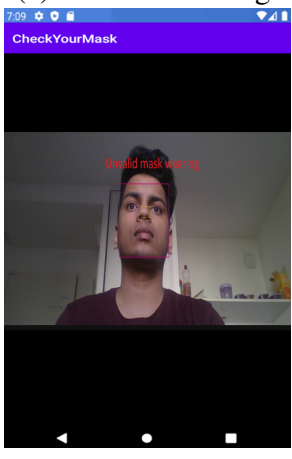

(c) Unvalid wearing.

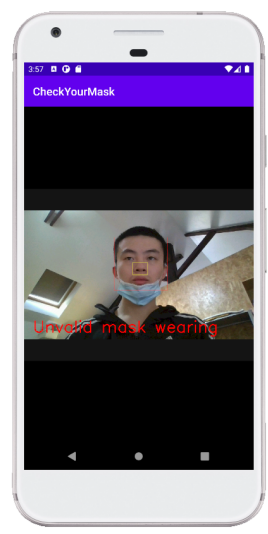

(e) Unvalid wearing.

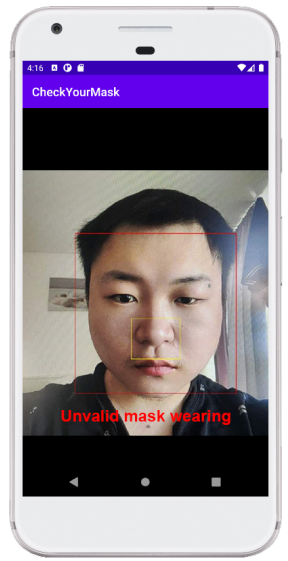

(g) Unvalid wearing.

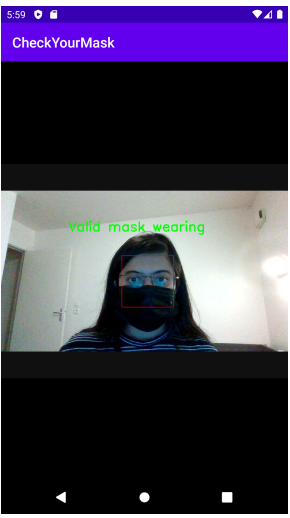

(b) Valid wearing.

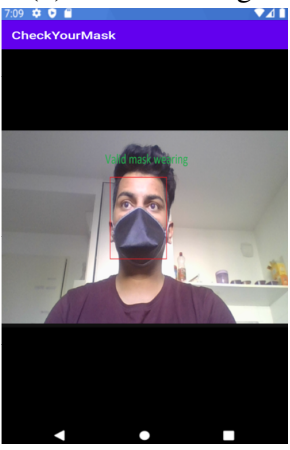

(d) Valid wearing.

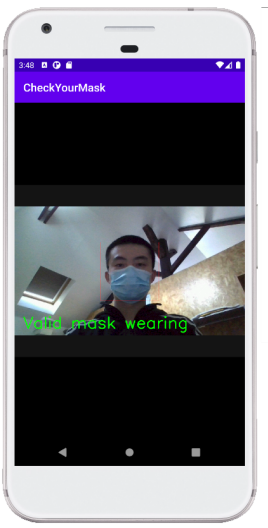

(f) Valid wearing.

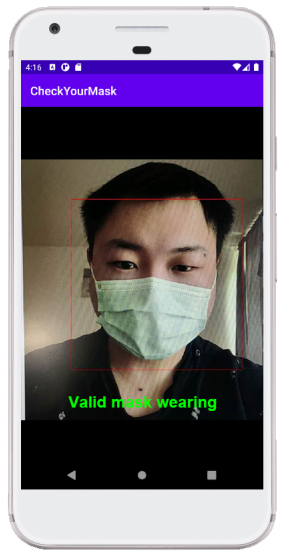

(h) Valid wearing.
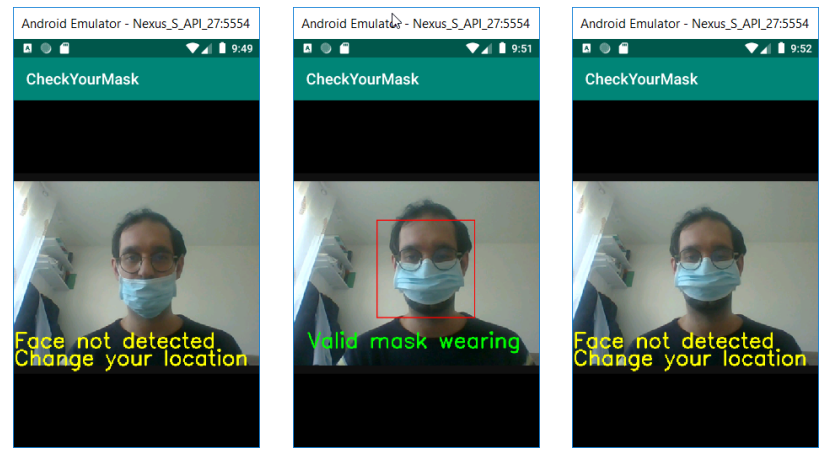

(a) Unprotected nose. (b) Unprotected chin. (c) Unprotected chin

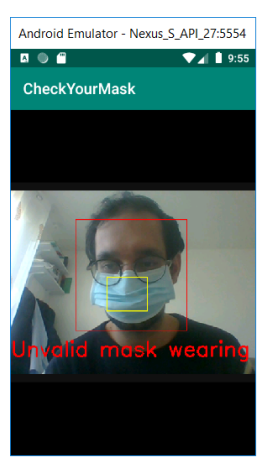

(d) Unprotected chin
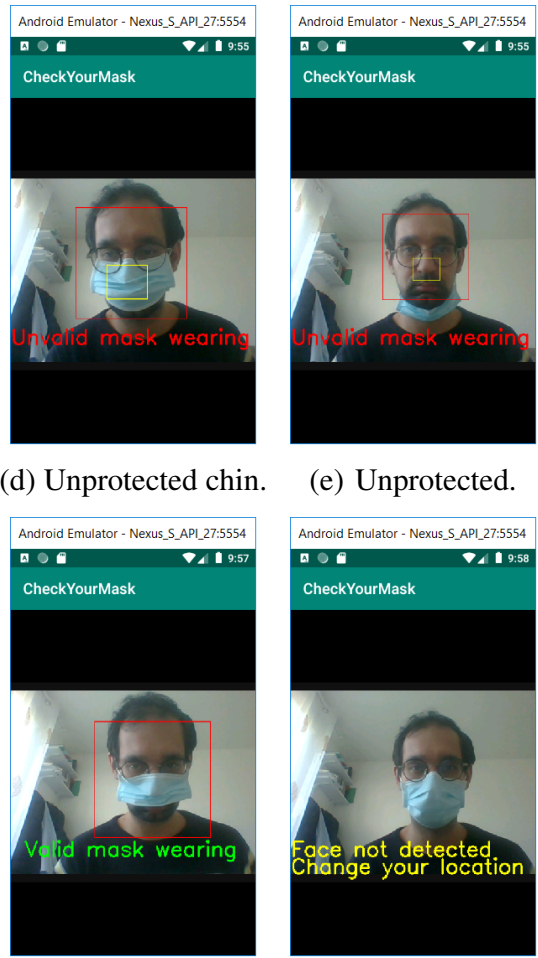

(e) Unprotected.
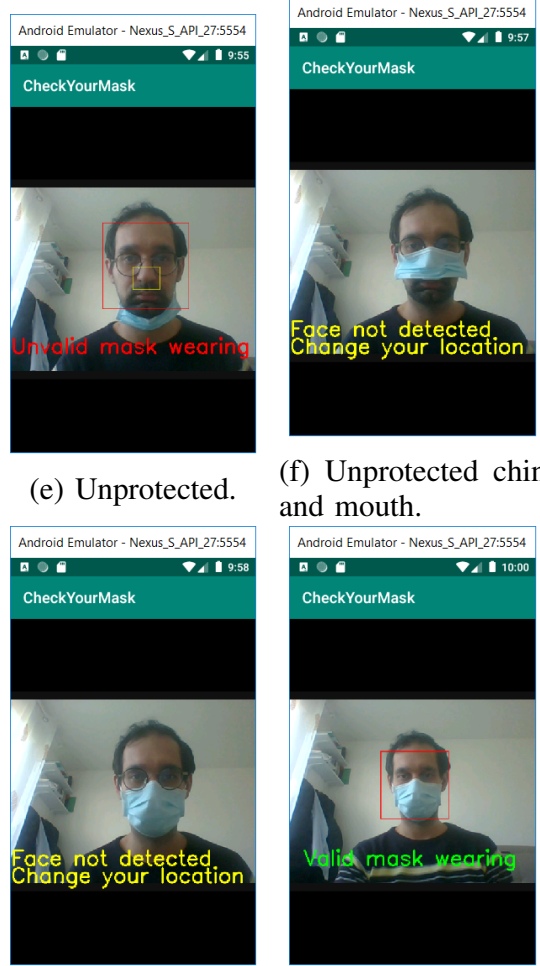

(f) Unprotected chin and mouth.

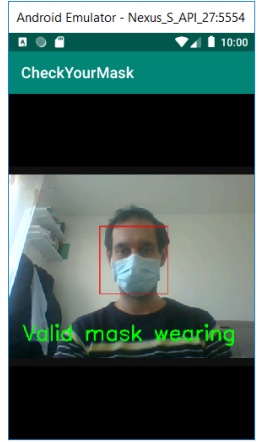

(g) Unprotected chin (h) Protected nose, (i) Protected nose, and mouth. mouth and chin. mouth and chin

Fig. 4: Test of configurations with respect to Figure 1. The user majoritarily wears glasses. The image background is heterogeous. A single-use blue face protection mask is used.

learning model with respect to specified face-feature categories or to correctly and incorrectly weared mask categories.

\section{ACKNOWLEDGMENTS}

The authors would like to thank the students Shraddha Shyam Shinde, Zhendong Qiang, Ashish Dharmendra Pal, Yingkui Chen, Parvez Gundumalli of the Master training in Information System, ESIGELEC School of Engineering, Rouen, France for their assistance in experiments.

Fig. 5: Tests of some users with different types of mask. 


\section{DISCLAIMER}

In no case the contributors of this work could be held responsible for any incident when using the designed application or masks.

\section{REFERENCES}

[1] "Frequently asked questions masque citoyen protection mutuelle ," 2020. https://cdn.nimbu.io/s/gd6c0r0/assets/1588090828970/FAQMasque-2020-04-28.pdf

[2] W. H. Organization, "Coronavirus disease (COVID-19) advice for the public: When and how to use masks," 2020. https://www.who.int/emergencies/diseases/novel-coronavirus2019/advice-for-public/when-and-how-to-use-masks

[3] N. Leung, D. Chu, E. Shiu, K. Chan, J. McDevitt, B. Hau, H. Yen, Y. Li, D. Ip, J. Peiris, W. Seto, G. Leung, D. Milton, and B. Cowling, "Respiratory virus shedding in exhaled breath and efficacy of face masks," Nature Medicine, Jan. 2020.

[4] S. Zhou, S. Lukula, C. Chiossone, R. Nims, D. Suchmann, and M. K. Ijaz, "Assessment of a respiratory face mask for capturing air pollutants and pathogens including human influenza and rhinoviruses," Journal of Thoracic Disease, vol. 10, pp. 2059-2069, 032018.

[5] M. Sande, P. Teunis, and R. Sabel, "Professional and home-made face masks reduce exposure to respiratory infections among the general population," PloS one, vol. 3, p. e2618, 022008.

[6] A. C. for Disease Control and A. U. Prevention Africa CDC, African Union), "How to wear a face mask correctly," 2020. https://africacdc.org/download/how-to-wear-a-face-mask-correctly/

[7] J. Bouteiller, "Coronavirus. comment bien porter son masque ? les conseils d'une infirmière de la métropole de lille," 2020. https://actu.fr/hauts-de-france/lille_59350/coronavirus-commentbien-porter-masque-conseils-dune-infirmiere_32651335.html

[8] A. S.-S. C. d'Ivoire, "Comment bien mettre son masque," 2020. https://www.facebook.com/110412877115436/photos/commentbien-mettre-son-masque/154573562699367/
[9] L. Colart, "Le port du masque: les gestes à faire et ne pas faire," 2020, https://www.lesoir.be/sites/default/files/dpistyles_v2/ena 16_9_in_line/2020/04/21/node_296003/27512244/public/2020/04/21/ B9723268640Z.1_20200421182927_000+GBLFTKA92.1-0.jpg?itok= vge-65yl1587734455 https://plus.lesoir.be/296003/article/2020-04-21/ le-port-du-masque-les-gestes-faire-et-ne-pas-faire

[10] M. Altaweel, "Using mobile phone data to limit the spread of COVID-19," 2020. https://www.gislounge.com/using-mobile-phonedata-to-limit-the-spread-of-covid-19/

[11] R. Robbins, "Can location data from smartphones help slow the coronavirus? facebook is giving academics a chance to try," 2020. https://www.statnews.com/2020/03/24/facebook-locationdata-coronavirus-spread/

[12] B. Deshayes, "StopCovid : nouvelle étape cette semaine pour l'application contre le coronavirus," 2020. https://www.linternaute.com/ actualite/guide-vie-quotidienne/2492203-stopcovid-nouvelle-etapecette-semaine-pour-1-application-contre-le-coronavirus/

[13] A. Rosebrock, "COVID-19: Face mask detector with opencv, keras/tensorflow, and deep learning," 2020. https://www.pyimagesearch.com/2020/05/04/covid-19-facemask-detector-with-opencv-keras-tensorflow-and-deep-learning/

[14] H. I. staff writters, "New technology allows identification through a mask," 2020. https://www.hospimedica.com/artificial-intelligence/ articles/294781567/new-technology-allows-identification-through-amask.html

[15] M. Castrillón Santana, O. Déniz Suárez, M. Hernández Tejera, and C. Guerra Artal, "Encara2: Real-time detection of multiple faces at different resolutions in video streams," Journal of Visual Communication and Image Representation, pp. 130-140, April 2007.

[16] P. Viola and M. J. Jones, "Robust real-time face detection," Int. J. Comput. Vision, vol. 57, no. 2, p. 137154, May 2004. https://doi.org/10.1023/B:VISI.0000013087.49260.fb

[17] R. Lienhart and J. Maydt, "An extended set of haar-like features for rapid object detection," vol. 1, 02 2002, pp. I-900. 